
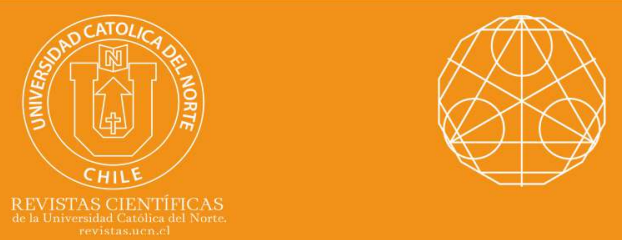

\title{
Generalizing unit-regular rings and special clean elements
}

Peter V. Danchev ${ }^{1}$ (10) orcid.org/0000-0002-2016-2336

${ }^{1}$ Institute of Mathematics and Informatics at the Bulgarian Academy of Sciences, Sofia, Bulgaria. iv danchev@math.bas.bg

\section{Abstract:}

As a strengthening of the definition of weakly clean rings, given by Šter in J. Algebra (2014), and as a common generalization of the classical unit-regular rings, we define and investigate the class of socalled weakly unit-regular rings as those rings $R$ for which, for every element $a \in R$, there exist a unit $u$ and an idempotent e such that $a-u-e \in(1-e) R a$ with $a R \cap e R$ $=\{0\}$. Some more exotic relationships with the well-known classes of clean, nil-clean and (strongly) $\pi$-regular rings are demonstrated as well. In particular, an elementwise extension of the so-called "special clean elements" by Khurana et al. in J. Algebra \& Appl. (2020) is also processed.

Keywords: Unit-regular rings; Clean rings; Special clean elements; Weakly clean rings; Weakly unit-regular rings.

MSC (2020): 16U99; 16E50; 13B99.

\section{Cite this article as (IEEE citation style):}

P. V. Danchev, "Generalizing unit-regular rings and special clean elements", Proyecciones (Antofagasta, On line), vol. 39, no. 5, pp. 1123-1135, Oct. 2020, doi: 10.22199/issn.0717-6279-202005-0069.

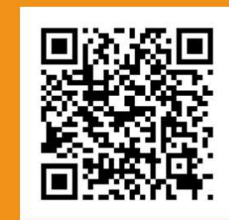

Article copyright: (C) 2020 Peter V. Danchev. This is an open access article distributed under the terms of the Creative Commons Licence, which permits unrestricted use and distribution provided the original author and source are credited. 


\section{Introduction and Conventions}

Everywhere in the text of the present article, all rings $R$ are assumed to be associative, containing the identity element 1 , which in general differs from the zero element 0 of $R$, and all subrings are unital (i.e., containing the same identity as that of the former ring). Our standard terminology and notations are mainly in agreement with [15]. For instance, $U(R)$ denotes the set of all units in $R, I d(R)$ the set of all idempotents in $R, \operatorname{Nil}(R)$ the set of all nilpotents in $R, J(R)$ the Jacobson radical of $R$, and $C(R)$ the center of $R$. For any unital subring $P$ of a ring $R$, we standardly set $K[R, P]=\left\{\left(r_{1}, r_{2}, \ldots, r_{n}, b, b, \ldots\right) \mid r_{i} \in R, b \in P, n \geq 1\right\}$. Then, it is easily checked that this set forms a ring under the standard operations of general addition and multiplication.

In the famous paper [25], generalizing the classical notion of boolean rings in which rings each element is an idempotent, it was introduced the fundamental class of (von Neumann) regular rings as those rings $R$ for which, for every $r \in R$, there is $x \in R$ such that $r=r x r$. On the other hand, in the subsequent important paper [8], it was considered a proper subclass of regular rings under the name unit-regular rings as being rings $R$ for which $r=r x r$ with $x \in U(R)$. If $r=r^{2} x$ with $r x=x r$, these rings are known as strongly regular rings. Good sources for examining these two ring classes are [9] and [24] as well as [5], [12] and [17], respectively.

A common generalization of unit-regular rings is nicely presented in [3] as the class of almost unit-regular rings was defined in the following manner: A ring $R$ is called an almost unit-regular ring, provided that, for any element $a \in R$, either $a$ or $1-a$ is unit-regular. It was shown there that there is an almost unit-regular ring which is not unit-regular (below this will be somewhat strengthened in Example 2.10 to the new point of view).

On the other vein, in the seminal paper [18], the class of clean rings was defined and studied intensively as those rings $R$ whose elements $r$ are a sum of a unit an idempotent, say $r=u+e$. If, in addition, $u e=e u$, these rings are called strongly clean. It is well known that (see, e.g., [19]) strongly regular rings are strongly clean as the reverse implication is wrong. Moreover, in [20] was constructed a unit-regular ring which is not strongly clean.

On the other side, as an expansion of the concept of clean rings, in [21] and [22] was introduced the class of weakly clean rings in the following 
manner: For every element $a \in R$, there exist a unit $u$ and an idempotent $e$ such that $a-u-e \in(1-e) R a$. It was shown there that there is a weakly clean non-clean ring.

On the other hand, it was long known from [2] that unit-regular rings $R$ can be characterized as a special kind of clean rings as follows: A ring $R$ is unit-regular if, and only if, for any $r \in R$, there exist $u \in U(R)$ and $e \in I d(R)$ such that $r=u+e$ with $r R \cap e R=\{0\}$. For a single element $r$, such a decomposition was recently called "special clean" in [14].

Our main purpose here is to develop the theory of unit-regular rings in the more attractive sense of weakly clean rings from [21], and thus we will rather naturally come to the definition of a weakly unit-regular ring. So, with all of the above at hand, we put into consideration the following new and slightly more precise notion.

Definition 1.1. A ring $R$ is said to be weakly unit-regular if, for each element $a \in R$, there are $u \in U(R)$ and $e \in I d(R)$ such that $a-u-e \in$ $(1-e) R a$ with $a R \cap e R=\{0\}$.

If, however, the existing idempotent in the representation of the element $a$ is unique, we shall say that the ring $R$ is uniquely weakly unit-regular.

Clearly, any unit-regular ring is weakly unit-regular. However, whether or not the converse implication is true, that is, there will exist a weakly unit-regular ring which is not unit-regular, is unknown yet (compare with Problem 2.12 stated below). Besides, the commutative, indecomposable, finite ring $\mathbf{Z}_{4}=\{0,1,2,3 \mid 4=0\}$ is manifestly not regular and also not weakly unit-regular, which follows easily by a direct check. We shall demonstrate in what follows that the classes of regular and weakly unit-regular rings are independent each to other (see, for instance, Corollary 2.6 listed in the sequel).

The motivation for writing up this paper is to discover some materialization between the already existing ring classes and the newly ones defined above. This is subsumed by the observation that this more restricted class of rings than the class of weakly clean rings possesses rather more exotic properties and also has a more complicated structure than it could be anticipated a priory.

\section{Preliminary and Main Results}

We foremost begin with a few useful facts and observations concerning weak unit-regularity. 
Proposition 2.1. If $R$ is a weakly unit-regular ring, then $J(R)=0$.

Proof. Let $a \in J(R)$. Then there exist $e^{2}=e \in R$ and $u \in U(R)$ such that $a-e-u \in(1-e) R a$ and $e R \cap a R=\{0\}$. As $(1-e) R a \subseteq J(R)$, one has that $e$ is a unit. Thus, $e=1$ and $a R=a R \cap R=\{0\}$. So, $a=0$, as desired.

In view of Proposition 2.1, for any ring $R$, the power series ring $R[[x]]$ and the upper triangular matrix $\operatorname{ring} \mathbf{T}_{n}(R)$ are manifestly not weakly unit-regular whenever $n \geq 2$.

Besides, it immediately follows from the above proposition that a weakly unit-regular ring is local (that is, each element is either a unit or lies in the Jacobson radical) exactly when this ring is a division ring. In fact, $R / J(R) \cong R$ is such a ring.

The last observation can be slightly enlarged thus:

Proposition 2.2. A weakly unit-regular ring $R$ is with only trivial idempotents if, and only if, $R$ is a division ring.

Proof. Assume that $R$ is weakly unit-regular and $\operatorname{Id}(R)=\{0,1\}$. For any $a \in R \backslash\{0\}$, one writes that $a-e-u \in(1-e) R a$ with $e R \cap a R=\{0\}$ for some $e \in I d(R)$ and $u \in U(R)$. As $a R \neq 0$, we have $e=0$. Thus $a-u \in R a$, which implies that $u \in R a$. Let $u=r a$ with $r \in R$. Then $u^{-1} r a=1$ and, therefore, $a\left(u^{-1} r\right)=1$ as it is a nonzero idempotent. So, $a \in U(R)$, and hence $R$ is a division ring. The converse is clear.

Recall that a ring $R$ is strongly regular if, for every $a \in R$, there exists $b \in R$ such that $a=a b a$ and $a b=b a$. It is well known that a ring is strongly regular if, and only if, it is abelian (i.e., all idempotents of the ring are central) regular if, and only if, it is reduced (i.e., without non-trivial nilpotents) regular (see, e.g., [9]). The next fact, which can be treated as a non-trivial extension of Proposition 2.2, is of some interest and importance.

Proposition 2.3. Let $R$ be a ring. Then the following three statements are equivalent:

(1) $R$ is strongly regular.

(2) $R$ is abelian weakly unit-regular.

(3) $R$ is uniquely weakly unit-regular. 
Proof. $\quad "(1) \Rightarrow(2) "$. As strongly regular rings are obviously unit-regular, the result follows at once.

$"(2) \Rightarrow(1) "$. Let $a \in R$. Since $R$ is weakly unit-regular, there exist $e^{2}=e \in R, u \in U(R)$ and $x \in R$ such that $a-e-u=(1-e) x a$ and $e R \cap a R=0$. As all idempotents are central, $a e=e a=0$. By multiplying $1-e$ on both sides of $a-e-u=(1-e) x a$, we have $u(1-e)=(1-x) a$. So $1-e=u^{-1}(1-x) a$. Write $s=(1-e) u^{-1}(1-x)$. Then $1-e=s a$, $s=(1-e) s$ and $s=$ sas. Note that as and $s a$ are idempotents. So, $a s=a(s a s)=a(s a) s=(s a)(a s)=s(a s) a=s a$. Observing that

$(s-e)(a-e)=(a-e)(s-e)=a s-a e-e s+e=a s+e=1-e+e=1$,

we obtain that $a-e:=v \in U(R)$. Since $a e=e a=0$, it readily follows that $a=a(1-e)=v(1-e)=(1-e) v$. It follows now that $a=a v^{-1} a$ and $a v^{-1}=v^{-1} a$. Thus $R$ is strongly regular, as asserted.

$"(1) \Rightarrow(3) "$. This implication is straightforward and so we leave the details to the interested reader.

$"(3) \Rightarrow(2)$ ". What we need to show is that $R$ is an abelian ring. To that purpose, given an arbitrary element $r \in R$ and an idempotent $e \in R$, one has the representations:

$$
-e=(-1)+(1-e)=(-1+(1-e) r e)+(1-e-(1-e) r e) .
$$

Clearly, $(1-e) r e$ is a nilpotent, so $-1+(1-e) r e$ is a unit as well as both $1-e$ and $1-e-(1-e) r e$ are idempotents as the latter satisfies the intersection requirement of Definition 1.1 pertaining to $-e$. Therefore, by the uniqueness of idempotents, $(1-e) r e=0$ leading us to the desired conclusion that $r e=$ ere.

Moreover, by a reason of some symmetry,

$$
e-1=-1+e=[-1+e r(1-e)]+[e-\operatorname{er}(1-e)],
$$

where $\operatorname{er}(1-e)$ being a nilpotent ensures that $-1+\operatorname{er}(1-e)$ is a unit, whereas $e-\operatorname{er}(1-e)$ is an idempotent and the elements $e-1$ and $e-\operatorname{er}(1-e)$ satisfy the intersection condition in Definition 1.1. Now, by the uniqueness of idempotents, we obviously have $e r(1-e)=0$ which means that $e r=e r e$. Combining this with $r e=e r e$, we unambiguously have that $e$ is central, because $r$ is arbitrary, as needed. 
It is worth mention that point (3) of the last result actually enlarges [6, Theorem 3.4], which refer to uniqueness in unit-regular rings, since strongly regular rings are exactly the subdirect product of division rings.

The next assertion, which can be viewed as a common extension of the previous Proposition 2.3, is somewhat quite curious. In fact, it is well known that the center of a (strongly) clean ring need not be clean (see, for example, [23]) but, however, the center of a regular ring is regular (and hence strongly regular) as well.

Proposition 2.4. If $R$ is a weakly unit-regular ring, then $C(R)$ is a strongly regular ring.

Proof. Letting $a \in C(R)$, there exist $e^{2}=e \in R, u \in U(R)$ and $x \in R$ such that $a=e+u+(1-e) x a$ with $e R \cap a R=\{0\}$. So, $a e=e a \in$ $e R \cap a R=\{0\}$. It follows that $a=(1-e) a=(1-e) u+(1-e) x a$. Multiplying the above equation by $a$ on the right, we obtain that $a^{2}=$ $(1-e) u a+(1-e) x a^{2}=u a+x a^{2}$ since $a \in C(R)$ and $a e=0$. Then, $a=u^{-1}(1-x) a^{2} \in R a^{2}=a^{2} R$. Thus, $a$ is a strongly regular element in $R$. By [19], one may write that $a=f v=v f$ for some $f^{2}=f \in R$ and $v \in U(R)$. Next we show that $f \in C(R)$. To that aim, for any $y \in R$, one sees that $f y(1-f)=v^{-1} a y(1-f)=v^{-1} y a(1-f)=0$, whence $f y=f y f$. Similarly, we deduce that $y f=f y f$. This proves that $f \in C(R)$. Writing $w=a-(1-f)=f v-(1-f)$, one deduces that $w \in C(R) \cap U(R)$ with the inverse $f v^{-1}-(1-f)$. Therefore, we finally conclude that $a=a f=w f=f w$ is strongly regular in $C(R)$, as desired.

Recall that a ring $R$ is said to be directly finite (or, in other words, Dedekind finite) if, for any two elements $a, b \in R$ satisfying $a b=1$, it follows that $b a=1$. It is principally known that unit-regular rings are directly finite. The following statement could be treated as an expansion of this crucial fact.

We are now ready to proceed by proving one of our chief results.

Theorem 2.5. Weakly unit-regular rings are directly finite.

Proof. For any element $a$ of such a ring $R$ we write that $a=u+e+$ $(1-e) x a$ with $a R \cap e R-\{0\}$, and let $a b=1$ for some $b \in R$. What suffices to prove is that $b a=1$. To that goal, having in mind that abe $=e \in$ $e R \cap a R=\{0\}$, we thereby have that $e=0$ and hence $a=u+x a$, that 
is, $a-x a=u$. Consequently, $a b=u b+x a b$, i.e., $1=u b+x$ and thus $u^{-1}(1-x)=b$. Therefore, $b a=u^{-1}(1-x) a=u^{-1}(a-x a)=u^{-1} u=1$, as wanted.

The next direct consequence is of some importance showing the independence of the classes of regular and weakly unit-regular rings.

Corollary 2.6. There exist regular rings (and thus a semiprimitive weakly clean ring) which are not weakly unit-regular.

Proof. Firstly, in virtue of [8] (see the discussion on p. 134 in [13] too), the ring of all linear transformations of an infinite dimensional vector space over a division ring is regular but not directly finite.

Secondly, in view of $[11$, p.13, Example 1] there is a regular ring which is not directly finite.

So, in both cases, we just apply Theorem 2.5 to conclude the claim as these two kind of rings violate the statement in that theorem.

Nevertheless, the next example gives some more light in constructing regular rings that are not weakly unit-regular.

Example 2.7. There exists a directly finite regular ring which is not weakly unit-regular.

Proof. In accordance with [11, p.14, Example 2], there is a regular ring $S$ which is directly finite but not unit-regular and which fails to be even weakly unit-regular. The verification of the latter claim we leave to the interested reader.

It was shown in [13, Corollary 7] that matrix rings of any size over unit-regular rings are again unit-regular. The same result for regular rings can be found in [16] and for clean rings in [10]. That is why, the question of whether or not the same claim holds for weakly unit-regular rings is of some interest.

As usual, for an arbitrary $n \in \mathbf{N}$, the letter $\mathbf{M}_{n}(R)$ denotes the full matrix ring over a ring $R$.

In regard to [21, Proposition 2.2] and expanding [14, Corollary 6.6], one may state the following:

Proposition 2.8. If the element $a$ of an arbitrary ring $R$ is special clean, then so does the element $\left(\begin{array}{cc}a & 0 \\ 0 & 0\end{array}\right)$

of a ring $\mathbf{M}_{2}(R)$. 
Proof. Write $a=e+u$ for some $e \in I d(R)$ and $u \in U(R)$ with $a R \cap e R=\{0\}$. Therefore, one can represent

$$
\left(\begin{array}{ll}
a & 0 \\
0 & 0
\end{array}\right)=\left(\begin{array}{cc}
e & (e-1) u \\
0 & 1
\end{array}\right)+\left(\begin{array}{cc}
u & (1-e) u \\
0 & -1
\end{array}\right)
$$

where it is not too hard to inspect directly that the first term is an idempotent, and the second term is a unit. Consequently, what remains to check is that the relation $\left[A \mathbf{M}_{2}(R)\right] \cap\left[E \mathbf{M}_{2}(R)\right]=\{0\}$ is true, provided $A=\left(\begin{array}{ll}a & 0 \\ 0 & 0\end{array}\right)$

and $E=\left(\begin{array}{cc}e & (e-1) u \\ 0 & 1\end{array}\right)$. To that aim, writing

$$
\left(\begin{array}{ll}
a & 0 \\
0 & 0
\end{array}\right)\left(\begin{array}{ll}
x_{1} & y_{1} \\
z_{1} & d_{1}
\end{array}\right)=\left(\begin{array}{cc}
e & (e-1) u \\
0 & 1
\end{array}\right)\left(\begin{array}{ll}
x_{2} & y_{2} \\
z_{2} & d_{2}
\end{array}\right),
$$

for some $x_{1}, x_{2} ; y_{1}, y_{2} ; z_{1}, z_{2} ; d_{1}, d_{2} \in R$, we just detect that

$$
\left(\begin{array}{cc}
a x_{1} & a y_{1} \\
0 & 0
\end{array}\right)=\left(\begin{array}{cc}
e x_{2}-(1-e) u z_{2} & e y_{2}-(1-e) u d_{2} \\
z_{2} & d_{2}
\end{array}\right)
$$

so that $z_{2}=0=d_{2}$ being immediately true implies that $a x_{1}=e x_{2} \in$ $a R \cap e R=\{0\}$ and that $a y_{1}=e y_{2} \in a R \cap e R=\{0\}$, substantiating the validity of our pursued relationship.

Remark 2.9. Trying to give some reminiscent of the last matrix presentation of $\operatorname{diag}(a, 0)$, and in conjunction with [3, Lemma 4.2], we will show that the above idea does not work properly further for $\operatorname{diag}(a, 1)$.

Indeed, for $a=e+u$ with $e \in \operatorname{Id}(R)$ and $u \in U(R)$ such that $a R \cap e R=$ $\{0\}$, one writes that

$$
\left(\begin{array}{ll}
a & 0 \\
0 & 1
\end{array}\right)=\left(\begin{array}{cc}
e & (e-1) u \\
0 & 1
\end{array}\right)+\left(\begin{array}{cc}
u & (1-e) u \\
0 & -1
\end{array}\right)+\left(\begin{array}{ll}
0 & 0 \\
0 & 1
\end{array}\right)
$$

where $B:=\left(\begin{array}{ll}a & 0 \\ 0 & 1\end{array}\right)$, and the other notations are as those stated in the preceding statement. Moreover, the last term $\left(\begin{array}{ll}0 & 0 \\ 0 & 1\end{array}\right)$ 
is obviously an idempotent.

However, the matrix $B$ cannot be represented of the form $B=E+$ $U+(1-E) X B$ for some $X=\left(\begin{array}{ll}x_{1} & x_{2} \\ x_{3} & x_{4}\end{array}\right) \in \mathbf{M}_{2}(R)$. In fact, $1-E=$ $\left(\begin{array}{cc}1-e & (1-e) u \\ 0 & 0\end{array}\right)$, so that $(1-E) X B$ contains a second row consisting of two zeros whence different to the matrix $\left(\begin{array}{ll}0 & 0 \\ 0 & 1\end{array}\right)$, as expected.

The next two constructions illustrate that the notions of almost unitregularity and weak unit-regularity are independent each to other.

Example 2.10. There exists an almost unit-regular ring which is not weakly unit-regular.

Proof. In [3, Example 2.8] was constructed an almost unit-regular ring which is not regular. We will demonstrate now that the so-stated rings is too not weakly unit-regular. In fact, let $F$ be a field, let $R=\mathbf{M}_{2}(F)$, and let $P=\left\{\left(\begin{array}{cc}a & b \\ 0 & a\end{array}\right) \mid a, b \in F\right\}$. Then $P$ is obviously a unital subring of $R$, and the ring $K[R, P]$ is almost unit-regular which is neither regular nor local. What we intend to do now is to show that $K[R, P]$ need not be weakly unit-regular as well. Indeed, consider the constant element

$$
A=\left(\left(\begin{array}{ll}
0 & 1 \\
0 & 0
\end{array}\right), \ldots,\left(\begin{array}{ll}
0 & 1 \\
0 & 0
\end{array}\right), \ldots\right) \in K[R, P] \text { by noticing that } P \text { is }
$$

not a weakly unit-regular ring. To see that, given $B=\left(\begin{array}{ll}0 & 1 \\ 0 & 0\end{array}\right) \in P$,

assume to contrary that there is an idempotent matrix $E$ and an invertible matrix $U$, both in $P$, such that

$$
B=E+U+\left(I_{2}-E\right) X B \text { with } B P \cap E P=\left\{0_{2}\right\}
$$

where $I_{2}$ and $0_{2}$ are the identity matrix and the zero matrix in $P$, respectively. Since by a plain computation we found that the only idempotents of $P$ are either $0_{2}$ or $I_{2}$, we have at once that $E=0$. Thus $B=U+X B$. Writing 


$$
\begin{gathered}
X=\left(\begin{array}{cc}
X_{1} & X_{2} \\
0 & X_{1}
\end{array}\right) \text {, we therefore deduce that } \\
U=B-X B=\left(\begin{array}{ll}
0 & 1 \\
0 & 0
\end{array}\right)-\left(\begin{array}{cc}
0 & X_{1} \\
0 & 0
\end{array}\right)=\left(\begin{array}{cc}
0 & 1-X_{1} \\
0 & 0
\end{array}\right)
\end{gathered}
$$

is not invertible by a direct inspection, a contradiction, showing also that $A$ is definitely not weakly unit-regular in $K[R, P]$, and so we are through.

Of great interest is to construct a weakly unit-regular rings which is neither regular nor clean. In that way, one states the following:

Conjecture: $A$ ring is unit-regular if, and only if, it is both regular and weakly unit-regular if, and only if, it is both clean and weakly unit-regular.

We end our work with the following challenging questions of some interest and importance.

It was proved in [1, Theorem 5] (see also [7, Lemma 3.9]) that any regular ring with bounded index of nilpotence is unit-regular. We shall now somewhat slightly expand this fact to the following one:

Problem 2.11. Are weakly unit-regular rings with bounded index of nilpotence unit-regular?

As we have seen above abelian (in particular, commutative) weakly unit-regular rings are themselves unit-regular (and thus strongly regular).

Problem 2.12. Is there a non-abelian weakly unit-regular ring which is not (almost) unit-regular?

We continue with the standard matrix question of whether or not the property "weak unit-regularity" is preserved by the full matrix ring. Notice that for unit-regular rings this question is completely settled in [13].

Problem 2.13. Suppose $R$ is a ring and $n \in \mathbf{N}$. Does it follow that the full $n \times n$ matrix ring $\mathbf{M}_{n}(R)$ is weakly unit-regular if, and only if, $R$ is weakly unit-regular?

We suspect that if $\mathbf{M}_{n}(R)$ is weakly unit-regular, then $R$ is also weakly unit-regular as well as if $R$ is weakly unit-regular, then $\mathbf{M}_{2}(R)$ is weakly unit-regular. For naturals $n \geq 3, \mathbf{M}_{n}(R)$ need not be longer weakly unitregular even if $R$ is so.

Our next query is the following: 
Problem 2.14. Characterize those rings $R$ for which, for every $a \in R$, there exist $u \in U(R)$ and $e \in I d(R)$ such that $a-u-e \in(1-e) R a$ or $a-u+e \in(1-e) R a$ with $a R \cap e R=\{0\}$.

In the sense of results from [4, Theorem 2.2] and [26], are these rings just weakly unit-regular?

\section{Acknowledgements.}

The author expresses his sincere thanks to Prof. Jian Cui from Anhui Normal University in Wuhu, China, for their valuable private communication on the present topic. Also, the author greatly acknowledges the partial support in writing up this research of the Bulgarian National Scientific Fund under Grant KP-06 N 32/1 of Dec. 07, 2019.

\section{References}

[1] G. Azumaya, "Strongly ח-regular rings", Journal of faculty of science, Hokkaido University. Series I. Mathematics, vol. 13, no. 1, pp. 34-39, 1954, doi: 10.14492/ hokmj/ 1530842562

[2] V. P. Camillo and D. Khurana, "A characterization of unit regular rings", Communications in algebra, vol. 29, no. 5, pp. 2293-2295, Apr. 2001, doi: 10.1081/AGB-100002185

[3] H. Chen, "On almost unit-regular rings", Communications in algebra, vol. 40, no. 9, pp. 3494-3506, Sep. 2012, doi: $10.1080 / 00927872.2011 .590953$

[4] A. Y. M. Chin and K. T. Qua, "A note on weakly clean rings", Acta mathematica hungarica, vol. 132, no. 1-2, pp. 113-116, Apr. 2011, doi: $10.1007 /$ s10474-011-0100-8

[5] P. V. Danchev, "Generalizing nil clean rings", Bulletin Belgian Mathematical Society -Simon Stevin, vol. 25, no. 1, pp. 13-28, 2018, doi: $10.36045 / \mathrm{bbms} / 1523412048$

[6] P. V. Danchev, "Uniqueness in von Neumann regular unital rings", Palestine journal mathematics, vol. 7, no. 1, pp. 60-63, 2018. [On line]. Available: https:/ / bit.ly/ 333Spaa

[7] P. V. Danchev and J. Šter, "Generalizing ח-regular rings", Taiwanese journal mathematics, vol. 19, no. 6, pp. 1577-1592, 2015, doi: 10.11650/ tjm.19.2015.6236

[8] G. Ehrlich, "Unit-regular rings", Portugaliae mathematica, vol. 27, no. 4, pp. 209-212, 1968. [On line]. Available: https:/ / bit.ly/ 3k2ZnmX 
[9] K. R. Goodearl, Von Neumann regular rings, 2nd ed. Malabar, FL: Krieger, 1991.

[10] J. Han and W. K. Nicholson, "Extensions of clean rings", Communication in algebra, vol. 29, no. 6, pp. 2589-2595, 2001, doi: 10.1081/ AGB-100002409

[11] D. Handelman, "Perspectivity and cancellation in regular rings", Journal algebra, vol. 48, no. 1, pp. 1-16, Sep. 1977, doi: 10.1016/ 0021-8693(77)90289-7

[12] R. E. Hartwig and J. Luh, "A note on the group structure of unit regular ring elements", Pacific journal mathematics, vol. 71, no. 2, pp. 449-461, 1977. [On line]. Available: https:/ / bit.ly/ 333XWNY

[13] M. Henriksen, "On a class of regular rings that are elementary divisor rings", Archiv der mathematik, vol. 24, pp. 133-141, Dec. 1973, doi: 10.1007/ BF01228189

[14] D. Khurana, T.-Y. Lam, P. P. Nielsen, and J. Šter, "Special clean elements in rings", Journal of algebra and its applications, vol. 19, no. 11, Art. ID. 2050208, 2020, doi: 10.1142/ S0219498820502084

[15] T.-Y. Lam, A first course in noncommutative rings, 2nd ed. New York, NY: Springer, 2001, doi: 10.1007/978-1-4419-8616-0

[16] T. Y. Lam, Exercises in classical ring theory, 2nd ed. New York, NY: Springer, 2003, doi: 10.1007/ b97448

[17] T. Y. Lam and W. Murray, "Unit regular elements in corner rings", Bulletin of the Hong Kong Mathematical Society, vol. 1, pp. 61-65, 1997. [On line]. Available: https:/ / bit.ly/ 3bvXA6u

[18] W. K. Nicholson, "Lifting idempotents and exchange rings", Transactions of the American Mathematical Society, vol. 229, pp. 269-278, 1977, doi: 10.1090/ S0002-9947-1977-0439876-2

[19] W. K. Nicholson, "Strongly clean rings and Fitting's lemma", Communications in algebra, vol. 27, no. 8, pp. 3583-3592, 1999. doi: 10.1080/ 00927879908826649

[20] P. P. Nielsen and J. Šter, "Connections between unit-regularity, regularity, cleanness, and strong cleanness of elements and rings", Transactions of the American Mathematical Society, vol. 370, no. 3, pp. 1759-1782, 2018, doi: 10.1090/ tran/ 7080

[21] J. Šter, "Corner rings of a clean ring need not be clean", Communications in algebra, vol. 40, no. 4, pp. 1595-1604, 2012, doi: 10.1080/ 00927872.2011.551901

[22] J. Šter, "Weakly clean rings", Journal algebra, vol. 401, pp. 1-12, Mar. 2014, doi: 10.1016/j.jalgebra.2013.10.034 
[23] J. Šter, "Examples of strongly clean rings", Communications in algebra, vol. 47, no. 11, pp. 4684-4696, 2019, doi: 10.1080/00927872.2019.15 88980

[24] A. A. Tuganbaev, Rings close to regular. Dordrecht: Kluwer Academic Publishers, 2002, doi: 10.1007/ 978-94-015-9878-1

[25] J. V. Neumann, "Examples of continuous geometries", Proceedings of the National Academy of Sciences, vol. 22, no. 2, pp. 101-108, Feb. 1936, doi: 10.1073/ pnas.22.2.101

[26] J.-C. Wei, "Weakly-Abel rings and weakly exchange rings", Acta mathematica hungarica vol. 137, pp. 254-262, 2012, doi: 10.1007/ s10474-012-0253-0 